\title{
AiMT
}

Advances in Military Technology

Vol. 11, No. 2 (2016), pp. 171-178

ISSN 1802-2308, eISSN 2533-4123

DOI 10.3849/aimt.01126

\section{Improvement of Low-Cost MEMS Gyroscope Characteristics by Data Filtering and Fusion}

\author{
M. Laššák ${ }^{1}$ and K. Draganová2* \\ ${ }^{1}$ Honeywell Technology Solutions, Control Systems CoE, Brno, Czech Republic, \\ ${ }^{2}$ Technical University of Košice, Faculty of Aeronautics, Košice, Slovakia.
}

The manuscript was received on 18 April 2016 and was accepted after revision for publication on 13 October 2016.

\begin{abstract}
:
Advancement of the MEMS technology at the end of the $20^{\text {th }}$ century allowed mankind to realize the things which had been unthinkable until then. Modern technological procedures have enabled a mass production of sensors and actuators of miniature dimensions with low purchase costs. The article deals with the fundamental principle of the MEMS gyroscopes as angular velocity sensors and with the necessary output data filtering and fusion with other inertial sensors to improve quality and precision of the angular velocity and position angles determination.
\end{abstract}

\section{Keywords:}

MEMS gyroscope, filtering, data fusion

\section{Introduction}

Gyroscopes, or angular velocity sensors, have been used in military and later also in civil aeronautics and marine for tens of years. The main part of the first rotational mechanic gyros, initially used on ships (still used in submarines), was a big and heavy rotating disc, momentum of which was maintained in a space. Derivative and directional gyros, mainly used in aeronautics, were obviously smaller in dimensions, lighter in weight, and the speed of a rotating disc was approximately $40000 \div 50000$ $\mathrm{rev} / \mathrm{min}$. Therefore precise manufacturing process was important, which lead to high costs. A little inaccuracy in mechanics could have resulted in significant gyro drift. An alternative to the mechanic gyros are expensive laser or fibre optic gyros, with excellent accuracy, and nowadays also miniature MEMS (micro-electro-mechanical systems) gyroscopes, initially used in mobile phones and amateur applications in robotics. Nowadays they are widely used together with accelerometers and magnetometers as

\footnotetext{
* Corresponding author: Technical University of Košice, Faculty of Aeronautics, Rampová 7 , SK-041 21 Košice, Slovakia. Phone: +421 556026 153,

E-mail: katarina.draganova@tuke.sk
} 
a part of inertial measurement units mainly for positioning, navigation and stabilization purposes not only in robotics, but also on boards of unmanned aerial vehicles (UAVs), which have been advantageously used in surveillance, monitoring, sensing, training and also battlefield applications. Long-term progress in manufacturing has resulted in continuously improving MEMS sensors' parameters that, after proper signal processing and data fusion, started to be used on boards of airplanes.

\section{MEMS Gyroscopes}

MEMS systems in general are systems with at least one dimension in micrometres. Considering the functional principles, MEMS gyroscopes can be divided into several categories. The best known and most widespread used are capacitive MEMS gyroscopes, using the change of a capacity as a physical measurement method. For the proper understanding of gyroscope principles in general, the Coriolis force understanding is essential [1-3]. Generally, the Coriolis effect is a phenomenon which acts upon any moving body in an independently rotating system and the Coriolis force can be computed as [4]:

$$
\mathbf{F}_{\mathrm{C}}=-2 m(\boldsymbol{\omega} \times \mathbf{v})
$$

where:

$F_{C}-$ Coriolis force,

$m$ - the mass of the moving body,

$\boldsymbol{\omega}$ - the angular velocity $[\mathrm{rad} / \mathrm{s}]$,

$\mathbf{v}$ - the velocity of the moving body in a rotating system.

As it can be seen from the Eq. (1), Coriolis force acts only on moving objects (v) in rotational frame $(\boldsymbol{\omega})$.

The consequences of the Coriolis force can be observed in nature - due to the Earth's rotation ocean currents and winds are deflected to the right in the Northern and to the left in the Southern hemisphere. Considering the ocean currents or winds, Coriolis force depends on the wind speed and also on the wind direction, and it varies with the latitude, too. Taking everything into account, Coriolis force changes from zero at the equator to a maximum at the poles. It does not curve only the winds and ocean currents, but it also affects for example paths of aircrafts, missiles or flying birds.

\subsection{MEMS Gyroscope's Functional Principle}

One axis gyroscope consists of two frames - inner and an outer one. The main part of the MEMS gyroscope is a moving object called "seismic mass", connected to the inner frame via miniature springs. The weight of the seismic mass is well-known during the manufacturing process. Gyroscope's electronic circuits consist of oscillator generating a very precise and stable driving frequency used for seismic mass oscillations. Considering capacitive type of a gyro, one driving capacitor's plate is mounted on the inner frame, while the other one on the seismic mass (the same capacitors are on the upper and bottom part of the seismic mass). When electrodes of driving capacitors are connected to the driving (oscillating) signal, the seismic mass is forced to oscillate periodically (for illustration - Fig. 1, seismic mass is moving up and down periodically). 


\subsection{Velocity of the Seismic Mass}

From the dimensions of the gyroscope construction and the driving frequency, the speed of seismic mass in each time frame can be determined. Considering Fig. 1 the seismic mass is oscillating at the same frequency and moving in $x$ axis. Velocity is changing during the period of oscillations. Starting in the bottom, velocity is $0 \mathrm{~m} / \mathrm{s}$, and as moving upward, velocity is increasing until the point where the forces (spring vs. capacitor force) are equal (in the middle) and then the velocity is decreasing. In the top position, the speed is again $0 \mathrm{~m} / \mathrm{s}$ and the movement in opposite direction starts.

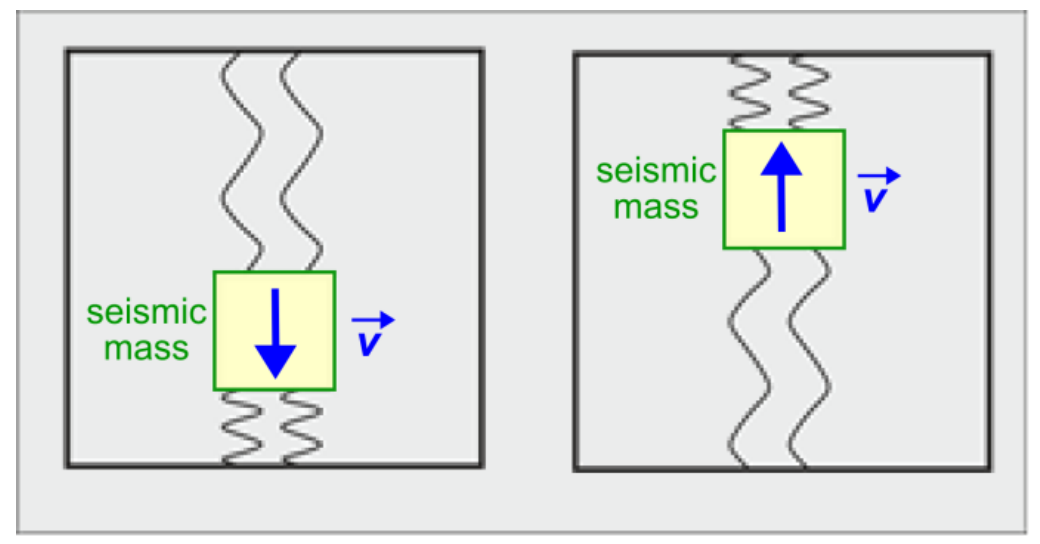

Fig. 1 Seismic mass in the inner frame

As the weight and the speed of seismic mass are known, angular velocity can be measured. When the object whose angular velocity is to be measured is rotating, the Coriolis force acts on the seismic mass. The direction of $\mathbf{F}_{\mathbf{c}}$ can be determined using Eq. (1).

While the seismic mass is oscillating, the direction of $\mathbf{F}_{\mathbf{C}}$ is changing periodically, too: considering right rotation when the mass moves upward, $\mathbf{F}_{\mathbf{C}}$ acts to the left side, causing the inner frame movement to the left. When the seismic mass moves downward, $\mathbf{F}_{\mathbf{C}}$ acts to the right side, causing the inner frame movement to the right. When object starts to rotate at some angular velocity, the inner frame movement can be detected. Sensing the inner frame movement is based on a simple capacitive principle: one capacitor's plate is mounted on the inner frame and the second one on the outer frame, the same capacitors are on both left and right sides, creating a differential capacitor. A displacement of the inner frame from the zero position (no angular velocity) is sensed as a change in capacity.

When the angular velocity is a constant, then the magnitude of the $\mathbf{F}_{\mathbf{C}}$ vector is a constant, too, and the direction of $\mathbf{F}_{\mathbf{C}}$ vector is changing in dependence on the velocity vector direction, causing the sensed capacity to be oscillating at the same frequency as the driving frequency. The value of capacity changes only in regard to the angular velocity. The direction of the angular velocity can be determined on the basis of the phase shift of two signals - of the driving signal (causing oscillations) and of the sensed signal (capacity change).

Fig. 2 a): When the object is rotating to the right side, the rotational vector has backward direction, the velocity upward direction, and Coriolis force acts to the left side. 
Fig. 2 b): Considering that the object is rotating still to the right side, the rotational vector has backward direction, but the velocity has downward direction, and Coriolis force acts to the right side.

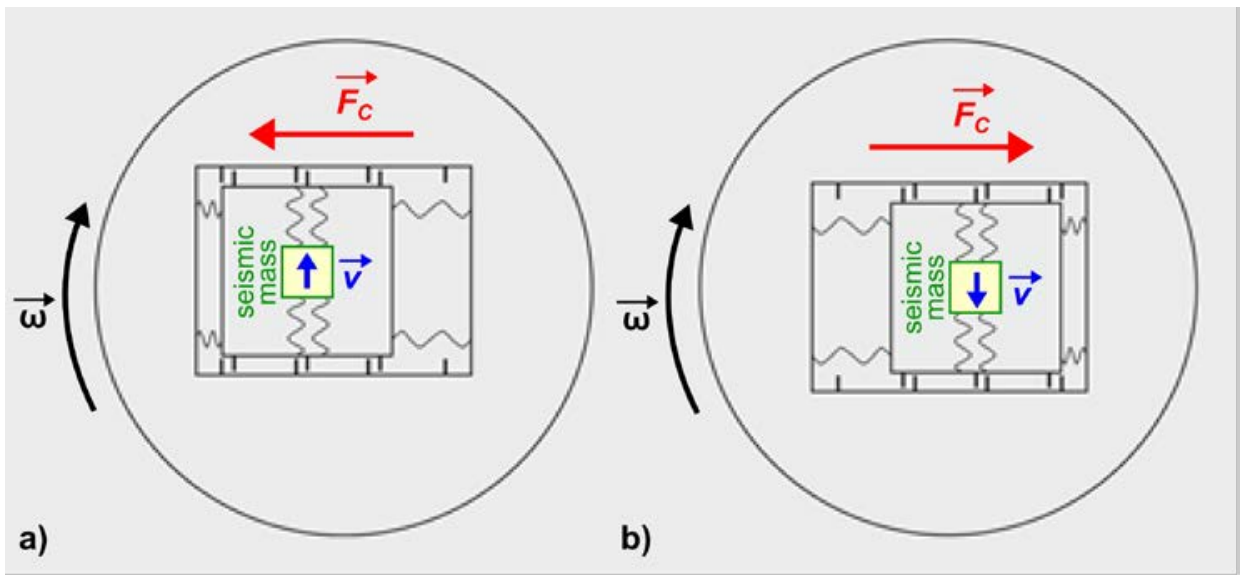

Fig. 2 Coriolis force acting on the inner frame

\subsection{Usage}

Gyroscopes as angular velocity sensors are mainly used in applications where the object's direction changes fast, so the angular velocities are significant - such for example in aircrafts, head trackers, camera gimbals, missiles.

Angular velocities are typically used in closed-loop control algorithms where angular velocities are used as a feedback for the improvement of the control algorithms performance. Another utilization possibility of gyroscopes is for the determination of position angles.

\subsection{Determination of Position Angles}

Gyroscopes can be used not only for the angular velocity measurements, but also the position angle can be computed by the angular velocity integration, using the following equation [4]:

$$
\boldsymbol{\alpha}=\int_{0}^{t} \boldsymbol{\Omega} \mathrm{d} t,
$$

where: $\boldsymbol{\Omega}$ - the angular velocity, $\boldsymbol{\alpha}$ - the angle, $\mathrm{d} t$ - the sampling period.

A disadvantage of the aforementioned angle computation principle is the fact that the mathematical integration of the angular velocity measurement noise causes a drift in the angle.

\section{Experiments and Results}

Measurements were performed using the 3-axis MEMS ITG 3200 gyroscope which is a part of an inertial measurement unit (IMU) with 9 DOF (Degrees of Freedom) connected to the mbed microcontroller which is based on the 32-bit NXP LPC1768 
microchip with the power supply stabilization (Fig. 3). All measurements were conducted using $100 \mathrm{~Hz}$ sampling frequency.

The sensed angular velocity data measured during the stationary measurement is illustrated in Fig. 4 The noise amplitude calculated as a standard deviation of the measured angular velocity achieved the value $\sigma=0.0473 \%$.

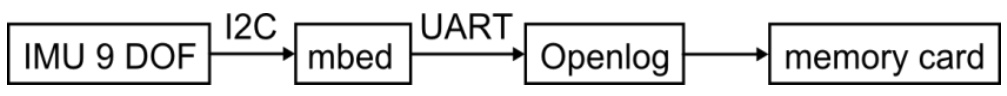

Fig. 3 Block scheme of measurement chain

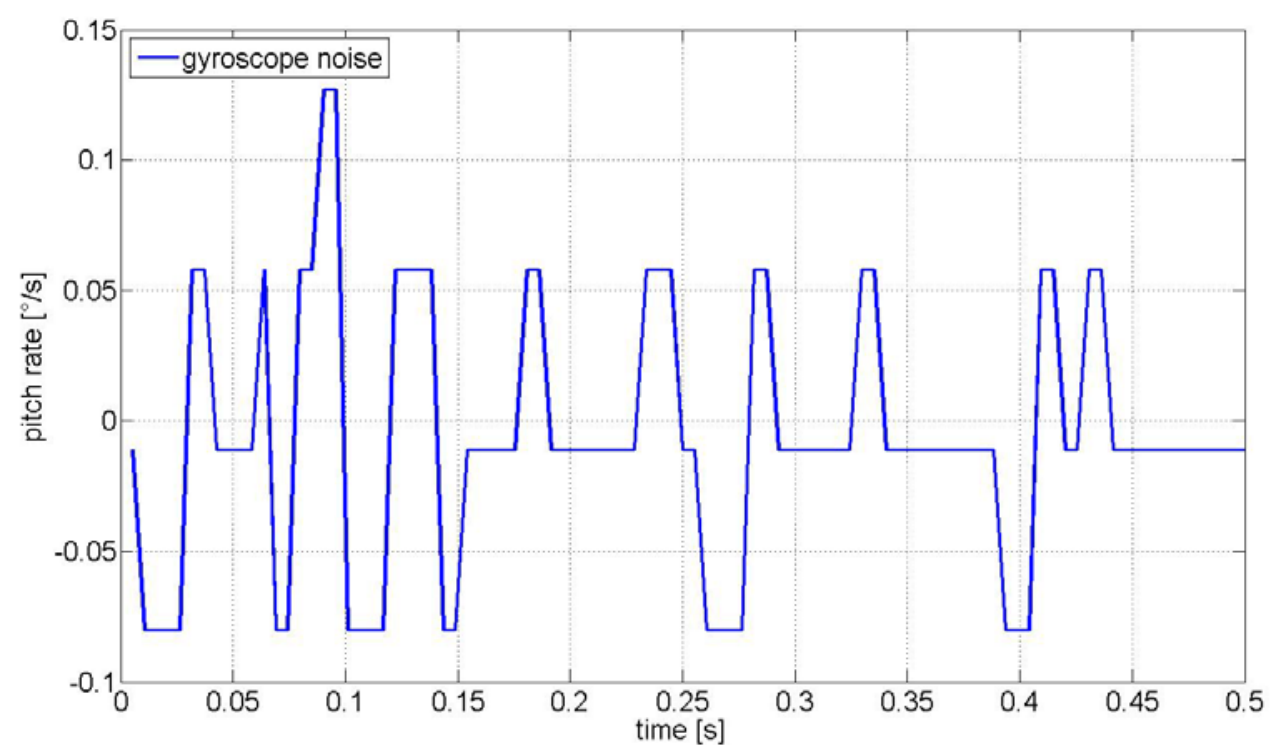

Fig. 4 Angular velocity noise

Using the Eq. (2), the angle was computed based on one-hour stationary measurement and it is shown in Fig. 5. Angular velocity noise during the integration process caused the drift of the angle that achieved approximately $15^{\circ}$ in 1 hour.

As Fig. 5 shows, using only MEMS gyroscopes data is not suitable to determine the long term angle, as the drift in the angle is unacceptable and it degrades the measurements. To achieve better results, sophisticated data-fusion and filtering algorithms have to be applied [5].

\section{Data Fusion}

For the gyro angle drift suppression, several methodologies have been used. Complementary filter based on data fusion of two sensors measuring the same value, with different noise in frequency domain [6], is a very simple and effective approach. For pitch and roll angle determination, the complementary filter uses data from an accelerometer (in our case for the measurements the ADXL 345 accelerometer was used, as part of the IMU with 9 DOF) affected by high frequency noise and gyro data (low 
frequency noise, causing drift). The complementary filter used in the mbed microcontroller was based on:

$$
\alpha(k)=b\left[\alpha(k-1)+\omega(k) T_{s}\right]+(1-b) \alpha_{A}(k),
$$

where:

$\alpha(k)$ - the pitch, (roll, yaw) in the $k$-th computational step,

$b$ - the constant of the filter (using our measurement chain, $b$ was tuned to 0.985 ),

$\omega(k)$ - the angular velocity in \% measured in the $k$-th computational step by the gyroscope,

$\alpha_{A}(k)$ - the angle determined by the accelerometer,

$T_{s}$ - the sampling period $\left(T_{s}=0.01 \mathrm{~s}\right)$.

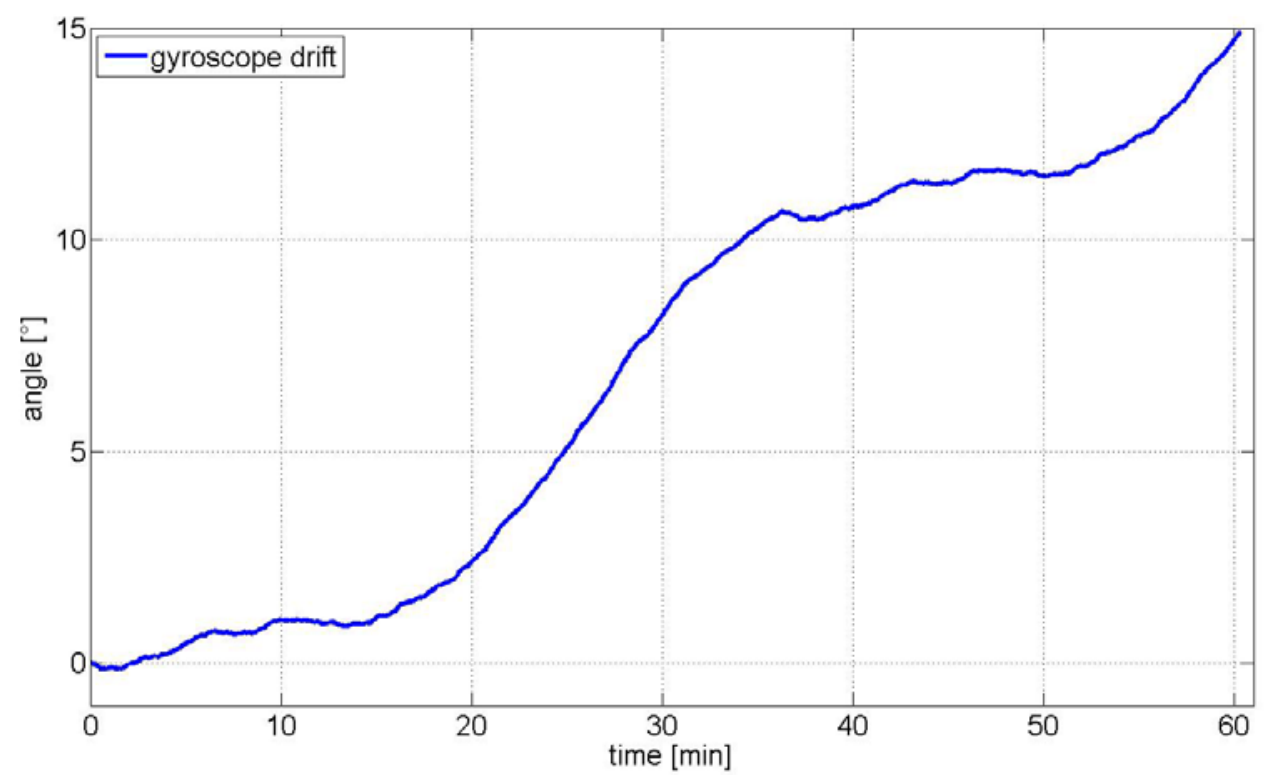

Fig. 5 Drift in computed angle

The data measured during the stationary measurement were pre-processed by the moving window median filter [7-9], and consequently by the moving window average filter for the output signal smoothing [10] (Fig. 6). The angle computed from the accelerometer output data was quite noisy, $\sigma=0.7872^{\circ}$. Afterwards the complementary filter was applied and the angle drift was eliminated. The standard deviation of the estimated pitch angle was $\sigma=0.1610^{\circ}$ (Fig. 6).

\section{Conclusion}

Gyroscopes are angular velocity sensors which have been used for many years in both the navy and aeronautics. However, due to the MEMS technology expansion in amateur robotics and low cost applications, the technology has become available also for general public. The functional principle of these gyroscopes is based on the Coriolis force. Nevertheless, the low-cost MEMS gyroscopes have poor noise parameters causing drift in the computed angle that makes them inapplicable for more precise or long-term measurements and that is the reason why many integration, data fusion and filtering techniques have been developed. The measurements presented in the 
paper have proved that modern digital signal processing algorithms and a simple complementary filter can effectively suppress the gyro angle drift and estimate the value of the angle even during the long-term measurements with excellent noise parameters and without drift, that allows MEMS sensors to be used in precise robotics for military and also civil applications.

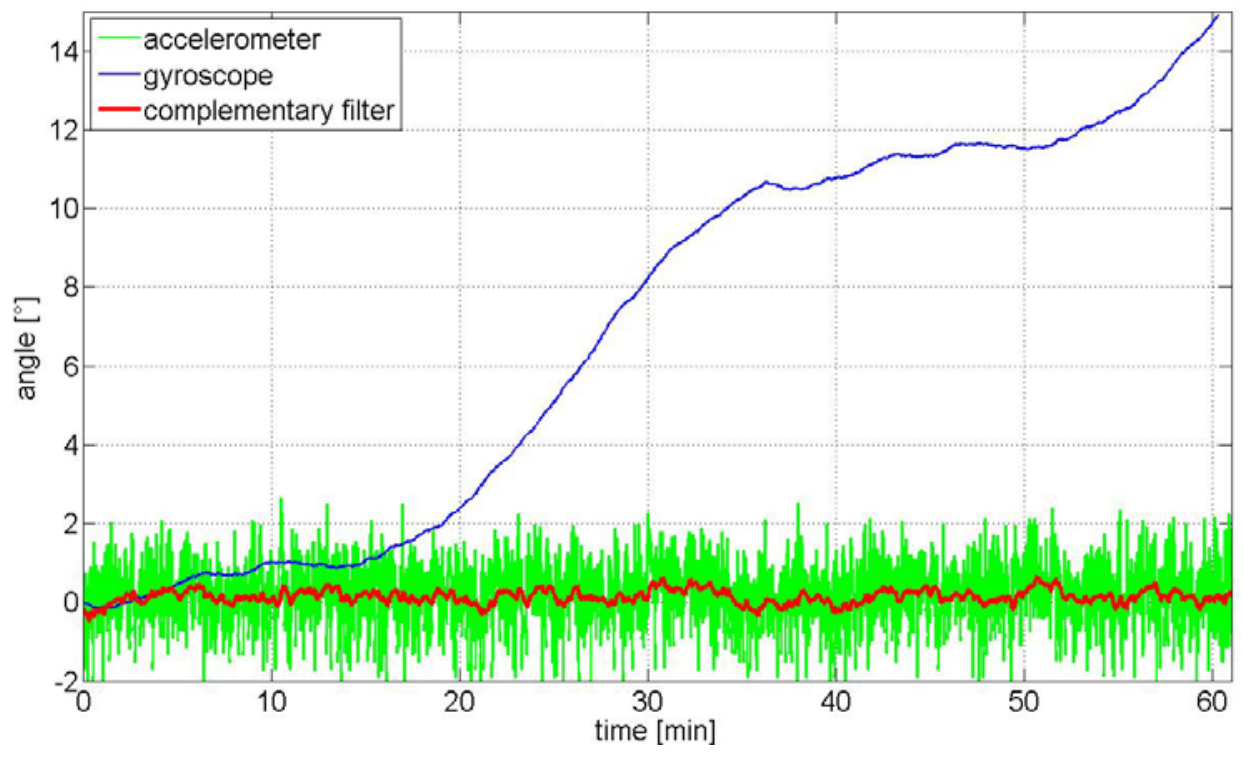

Fig. 6 Complementary filtered data

\section{Acknowledgement}

This work was supported by the Slovak Research and Development Agency under contract No. APVV 0266-10 and Scientific Grant Agency of the MESRaS SR and SAS under contract No. VEGA 1/0201/16.

\section{References}

[1] LAŠŠÁK, M. Controlling of multisensor system (in Slovak). [PhD Thesis]. Košice: Technical University of Košice, 2014. 125 p.

[2] BARBOUR, N. and SCHMIDT, G. Inertial sensor technology trends. In IEEE Sensors J., 2001, vol. 1, no. 4, p. 332-339. DOI: 10.1109/7361.983473.

[3] YAZDI, Y., AYAZI, F. and NAJAFI, K. Micromachined inertial sensors. In Proc. IEEE, 1998, vol. 86, no. 8, p. 1640-1659. DOI: 10.1109/5.704269.

[4] SOTÁK, M., SOPATA, M., BRÉDA, R., VÁCI, L. and ROHÁČ, J. Navigation System Integration. Košice: Róbert Bréda, 2006. 343 p.

[5] VAISPACHER, T., ANDOGA, R., BRÉDA, R. and ADAMČÍK, F. Application of linearized Kalman filter in integration of navigation systems. In CINTI 2015. Danvers: IEEE, 2015, p. 79-84. DOI: 10.1109/CINTI.2015.7382898. 
[6] COLTON, S. The Balance Filter: A Simple Solution for Integrating Accelerometer and Gyroscope Measurements for a Balancing Platform [on line]. Cambridge: Massachusetts Institute of Technology. [cited 10-10-2015]. Available from: <http://d1.amobbs.com/bbs_upload782111/files_44/ourdev_665531S2JZG6.pdf>.

[7] EVANS, JR. Running median filters and a general despiker. Bulletin of the Seismological Society of America, 1982, vol. 72, no. 1, p. 331-338.

[8] STONE, CD. Application of median filtering to noisy data. Canadian Journal of Chemistry, 1995, vol. 75, no. 10, p. 1573-1581.

[9] SMITH, SW. The scientist and Engineer's Guide to Digital Signal Processing Moving Average Filters. San Diego: California Technical Publishing, 1997, p. 277-284.

[10] LAŠŠÁK, M., DRAGANOVÁ, K. and PRASLIČKA, D. Application of moving window median filter for UAV control. In: MOSATT 2013: Proceedings of the International Scientific Conference Modern Safety Technologies in Transportation. Košice: Perpetis, 2013, p. 149-154. 